\title{
UNUSUAL PRESENTATION OF TUBERCULOSIS IN ORAL CAVITY: A CASE REPORT
}

Shweta Sharma ${ }^{1}$, Anjali Wadhwa², Anand Kumar Sajjan³, Gaurav Shah ${ }^{4}$

\section{HOW TO CITE THIS ARTICLE:}

Shweta Sharma, Anjali Wadhwa, Anand Kumar Sajjan, Gaurav Shah. "Unusual Presentation of Tuberculosis in Oral Cavity". Journal of Evolution of Medical and Dental Sciences 2014; Vol. 3, Issue 13, March 31;

Page: 3378-3383, DOI: $10.14260 /$ jemds/2014/2294

ABSTRACT: Tuberculosis is a chronic disease which is still prevalent in developing countries like India. Although it is mainly a pulmonary disease with extra pulmonary manifestations, oral manifestations are not common. We are presenting a case in which tuberculous ulcers were present in oral cavity in more than one location and resembled carcinoma. Medical history is very much important and conditions which may have similar clinical pictures should be ruled out before.

KEYWORDS: Tuberculosis, Oral cavity, Ulcers, Pulmonary.

INTRODUCTION: Tuberculosis is a chronic infectious granulomatous disease caused by acid-fast mycobacterium tuberculosis, Mycobacterium bovis and other atypical mycobacterium. Extrapulmonary involvement in tuberculosis is rare, accounting for about 10 to $15 \%$ of all cases. ${ }^{1}$ The incidence of oral tuberculosis in preantibiotic era was less than $1 \%$ that of pulmonary tuberculosis. Thirty percent to sixty percent of all cases occur in developing countries. The plausible reasons for this increase may be: lack of public health efforts to control TB following its elimination; the epidemic of HIV infection; increase in poverty and the large number of people in crowded shelters; and development of multi drug resistant species of bacteria.

The clinical presentation of TB may take many forms and frequently overlooked in the differential diagnosis of oral lesions. The purpose of this article is to report a case of secondary TB at the oral mucosa which is a rare location for TB infection. TB may be either a primary or a secondary infection.2, 3 Primary oral TB lesions are extremely rare, generally occur in young patients and are associated with cervical lymphadenopathy. Primary lesions remain painless in the majority of cases and are manifested in immunocompromised conditions (e.g., HIV infection) more frequently than its secondary counterpart.4

Secondary lesions are more common and are mostly seen in older people. The oral manifestations of TB are superficial ulcers, patches, indurated soft tissue lesions or even lesions within the jaw that may be in the form of TB osteomyelitis or simple bony radiolucency.

Of all these oral lesions, the ulcerative form is the most common. It is often painful, with no caseation of the dependent lymph nodes and to emphasize the importance of early diagnosis to lessen the risk of exposure to an infected patient's contacts. ${ }^{2,3}$

CASE REPORT: A 55 year old man reported to department of Oral and Maxillofacial Surgery with the complain of an ulcer in the mouth which was present for last 7 months and had failed to heal despite the administration of antibiotics and other topical applicants. It was gradually increasing in size and was painful, eventually restricting him to soft diet.

His past medical history revealed history of pulmonary TB 20 years back, for which he took treatment but he did not complete the full course of antibiotics. He was a chronic smoker and was 
unable to give up the habit. The patients' appearance was cachectic and had noted progressive loss of weight and had anorexia. Physical examination did not reveal any extra oral abnormality. His sub mandibular lymph nodes were enlarged. Measuring about $1.5 \times 1 \mathrm{~cm}$, oval in shape, rubbery in consistency, mobile and non-tender on palpation. Intra oral hygiene was graded as poor with significant halitosis. Soft tissue examination revealed a single discrete ulcer of $2.5 \times 2.5 \mathrm{~cm}$ in diameter on right buccal mucosa. (fig1)

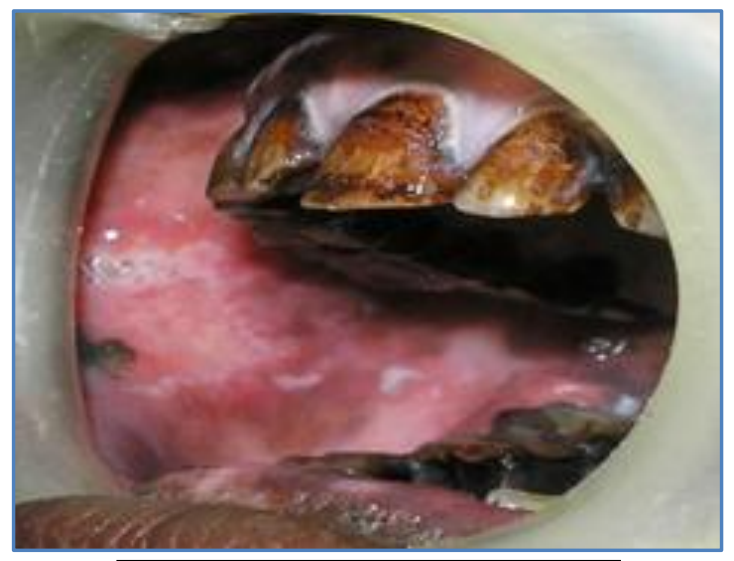

Fig 1: Tuberculous ulcer over right buccal mucosa

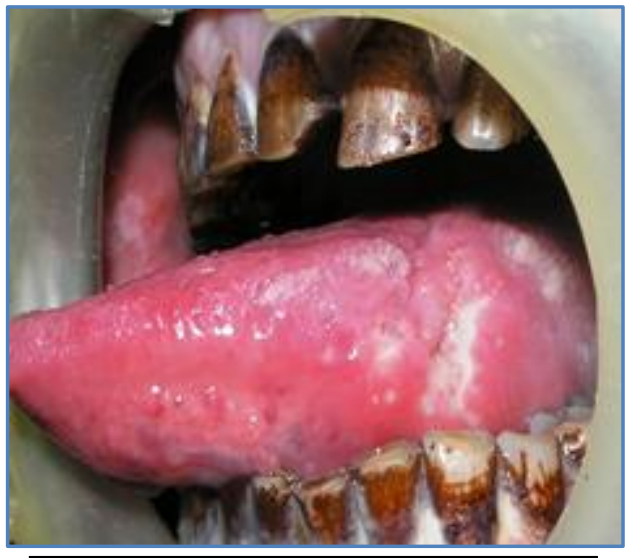

Fig 2: Tuberculous ulcer over left lateral border of tongue

The ulcer was bordered by well-defined margins. On palpation, the ulcer was tender with indurated margins. He also presented with a non-healing ulcer measuring about $2 \times 4 \mathrm{~cm}$ on the left lateral border of the tongue simultaneously (fig2) .The ulcer had irregular undermined borders and was non tender on palpation with minimal induration. The other mucosal surfaces in the oral cavity were normal. Panoramic radiograph did not reveal any abnormality in the maxillofacial region.

A complete general examination revealed no other contributory abnormalities. Hence a differential diagnosis of malignant neoplasm was arrived, possibly a squamous cell carcinoma, and so an incisional biopsy of the lesion was performed under local anesthesia. The diagnostic work up included serum analysis for HIV which turned out to be non- reactive.Blood tests were within normal limits except for low $\mathrm{Hb}(8.8 \mathrm{gms} \%)$, raised platelet count $(4,17,000)$ and raised ESR $(95 \mathrm{~mm} / \mathrm{hr}$.). Histopathological examination of incisional biopsy specimen of affected mucosa showed compression of fibrocellular connective tissue with few muscle bundles, hemorrhagic areas and an area suggestive of adipose tissue.

The connective tissue was comprised of diffuse and dense chronic inflammatory cells predominantly lymphocytes with 4-5 areas showing aggregates of giant cells with nuclei placed peripherally in the form of Horse shoe pattern in few cells. A cellular eosinophilic material suggestive of necrosis surrounded by fibrosis was also evident. One bit of tissue showed normal squamous epithelial tissue infiltrated by inflammatory cells. Thus granulomatous inflammation containing Langhans type giant cells raised the possibility of granulomatous infection. FNAC of submandibular lymph node showed multiple confluent and discrete necrotizing granulomas.

Immunological investigation ELISA on patient serum with detection of IgM, IgG \& IgA antibody antimycobacterium tuberculosis and anti-atypical mycobacterium was performed and revealed positive result. 


\section{CASE REPORT}

Chest $\mathrm{x}$ - ray shows fibro -calcific foci in both upper lobes with evidence of active lesion cavernous (cavity) in right perihilar region suggestive of Pulmonary Tuberculosis (fig. 3).

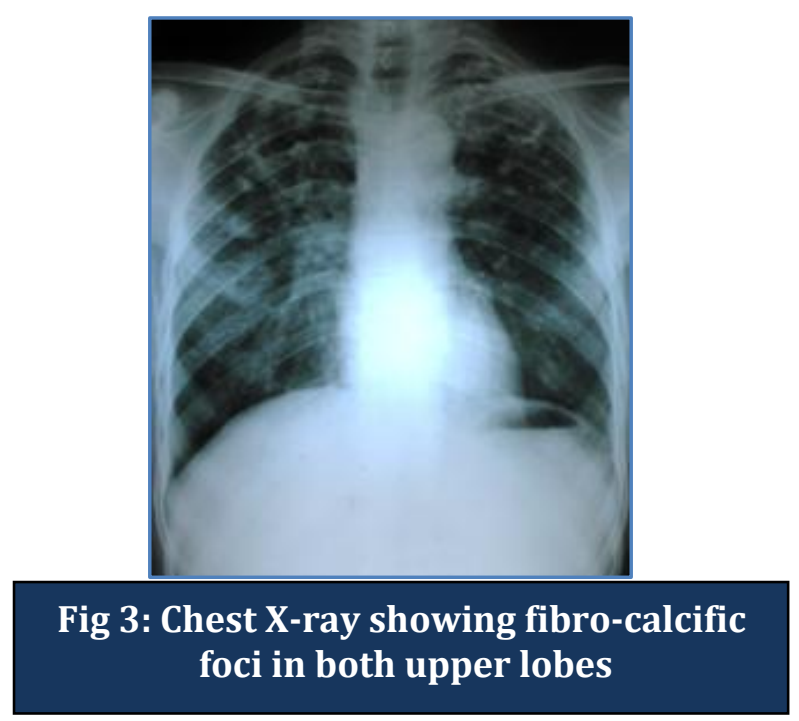

On contrast CT, bilateral opacification in the upper lobes a $2 \mathrm{~cm}$ cavity with a regular border in the superior segment of the left lower lobe and nodular opacity on the same segment of the same size were present. (Fig. 4)

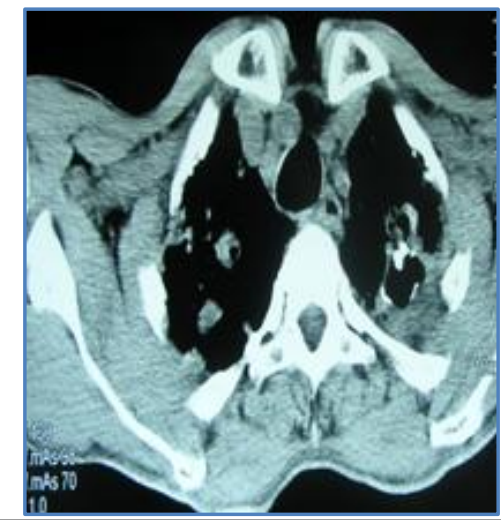

\section{Fig 4: CT showing cavity with a regular border in the superior border segment of the left lower lobe}

A diagnosis of pulmonary TB was confirmed by the culture of the mycobacterium TB from sputum samples. Following which the patient was managed with ATT regimen by physician. One month after beginning ATT the lesion healed and showed complete resolution. The patient is on follow up for 4 months without showing any recurrence.

DISCUSSION: Tuberculous infection can either be primary or secondary; the primary form affects the lungs lymph nodes, meninges, kidneys, bones, and skin. The secondary form occurs due to spread of infection from other parts of body5. Two types of presentation are seen in oral TB: Primary lesions occurring as a result of direct inoculation of oral tissues and secondary infection occurring due to hematogenous or lymphatic spread or from direct extensions from neighboring structures 6 .The topic 
is of concern in the India, where there are lack of public health efforts to control TB, inadequate nutrition in poor socioeconomic population, epidemic of HIV infection, inadequate supply of vaccine being produced, reduced availability of antitubercular drugs and increased incidence of multi drug resistant strains of Mycobacterium TB .

And also with increasing number of TB cases, un usual forms of the disease in the oral cavity are most likely to occur and be misdiagnosed and hence gaining control over TB is becoming all the more difficult. The mechanism of primary inoculation is not known. However, it is thought that the mycobacterium is inoculated directly into the oral mucosa. The intact oral mucous membrane presents a natural resistant to invasion by mycobacterium. This has been attributed to the cleansing action of saliva, the presence of salivary enzymes, tissue antibodies, oral saprophytes thickness of the protective epithelial covering. Any break loss of this barrier, which may be the result of trauma, inflammatory conditions, tooth extraction, or poor oral hygiene, may provide a route of entry for the organism.

In cases of secondary lesions with respect to the route of infection of the bacilli in the oral cavity, it is generally regarded that pulmonary bacilli are transmitted to lesions via Lymphatic or hematogenous routes. Both systemic and local factors play a role in incidence of oral lesions. Systemic factors include lowered host resistance and increased virulence of the organisms. ${ }^{7}$ Local factors comprises poor oral hygiene, local trauma, chronic inflammation, tooth eruption, extraction sockets, periodontal disease, carious teeth with pulp exposure and presence of lesions like leukoplakia, dental cysts, dental abscesses, and jaw fractures. Any breach in the mucosal lining predisposes toward oral involvement. ${ }^{5}$

Primary form of tuberculous oral lesions usually affects the gingiva and mucobuccal folds. An inflammatory focus adjacent to teeth or teeth extraction sites has also been reported. In addition, primary lesions are often associated with enlarged cervical lymph nodes. The secondary form is more frequent in middle-aged and older persons and involves mainly the tongue and hard palate. ${ }^{9}$

Neck TB is less infective. If the amount of mycobacterium in the ulcerative oral TB lesion is high enough, there is the possibility that more lesions could be induced and disseminated to other individual via the saliva5. For patients with pulmonary TB, close contacts are those sharing a house and are most at risk. Occasionally a contact at work is close enough to be equivalent to a household contact.

Casual contacts are most occupational contacts and need only be examined if they are unusually susceptible to infection, such as immunocompromised adults. All staffs in regular contact with patients are at potential risk of contracting TB. The route of infection of the bacilli in the oral cavity is generally regarded that pulmonary bacilli are transmitted to secondary lesions via lymphatic or hematogenous routes. ${ }^{10}$

The chronic ulcerative form is the most common of these oral lesions. In the presence of chronic oral ulcer, the differential diagnosis usually considered are malignancies, sarcoidosis, syphilis, aphthous lesion, mycotic infections and traumatic injuries. However TB is often overlooked by diagnosis practitioner. ${ }^{10}$

A number of HIV infection patients were identified in India during the period of Jan 2010March 2010 out of which one third had co-infection with TB. TB can be asymptomatic in HIV patients. A careful recording of the medical history should be performed. In addition some opportunistic 
infection notably TB can appear even when patient are receiving highly active anti retro viral therapy as HIV viral loads fall and CD4 T cell counts improve.

CONCLUSION: As with other tuberculous lesions of the oral cavity they can resemble carcinoma or the two can coexist.This emphasize the importance of an early diagnosis and bacteriological tests to differentiate between the lesions. Because the clinical picture of oral TB in AIDS patients may be atypical and unusual, general clinicians, dentist and Otolaryngologist must keep alert for early diagnosis and treatment .An early diagnosis and treatment will usually result in a complete cure. TB chemotherapy is very successful in the treatment of oral cavity TB. Although occupational infection is uncommon, pre-employment screening should be carried out with details of previous BCG vaccine obtained and presence or absence of a BCG. A heaf test is only necessary in the absence of a BCG scar.

All health care workers should be protected by BCG vaccine and should be checked for titre if booster doses required. The known cases should be treated with improved anti -TB chemotherapy for prolonged period.

No matter whether primary or secondary oral TB in nature, early detection, diagnosis and treatment are of the outmost importance. Oral lesion and concurrent pulmonary lesions also alert the oral physician to consider systemic disease so that confirmatory diagnostic studies can be performed.

\section{REFERENCES:}

1. Wen-Chen Wang, Jin -Yi Chen, , Yuk-Kwan Chen et al. Tuberculosis of head and neck: a review of 20 cases, Oral Surgery, Oral Medicine, Oral Pathology, Oral Radiology. Vol. 107 No.3 March 2009.

2. Bahar S, Zeytinoglu M, Tuncay U, Unnal T. Oral mucosal ulceration: A manifestation of previously undiagnosed pulmonary tuberculosis. J Am Dent Assoc, Vol 135, No 3, 336-340.

3. Eng HL, Lu SY, Yang CH, Chan WJ. Oral tuberculosis, Oral Surgery, Oral Medicine, Oral Pathology, Vol. 81 No44 April 1996.

4. Daley CL, Small PM, Schecter GF, Schoolnik GK, Mc Adam RA, Jacobs WR Jr, Hopewell PC. An outbreak of tuberculosis with accelerated progression among persons infected with the human immunodeficiency virus. N Eng J Med 1992; 320:231-5.

5. Kataria SP, Singh S, Parul T, Kumar S, Singh G. Primary tuberculosis of the tongue in an immunocompetent patient: A case report Asian Pacific J Trop Dis 2012;2:78-9.

6. Mahajan S, Srikant N, George T. Atypical Presentation of Oral Tuberculosis Ulcer. New York State Dent J 2007; 73:48-50.

7. Ebenezer J, Samuel R, Mathew GC, Koshy S, Chacko RK, Jesudason MV. Primary oral tuberculosis: Report of two cases. Ind J Dent Res 2006; 17:41-4.

8. Kumar S, Sen R, Rawal A, Dahiya RS, Dalal N, Kaushik S. Primary Lingual Tuberculosis in Immunocompetent Patient: A Case Report. Head Neck Pathol 2010;4:178-80

9. Erbaycu AE, Taymaz Z, Tuksavul F, Afrashi A, Güçlü SZ. "What happens when oral tuberculosis is not treated?" Monaldi Arch Chest Dis 2007; 67:116-8.

10. Caken, Z Mutlu, A Ozsoz et al. Tuberculosis of oral mucosa. Monaldi Arch Chest 2001; 56: 4, 315317. 


\section{AUTHORS:}

1. Shweta Sharma

2. Anjali Wadhwa

3. Anand Kumar Sajjan

4. Gaurav Shah

\section{PARTICULARS OF CONTRIBUTORS:}

1. Professor, Department of Oral and Maxillofacial Surgery, NIMS Dental College, Jaipur.

2. II ${ }^{\text {nd }}$ Year Post Graduate, Department of Oral and Maxillofacial Surgery, NIMS Dental College, Jaipur.

3. Senior Lecturer, Department of Oral and Maxillofacial Surgery, NIMS Dental College, Jaipur.
4. Reader, Department of Oral and Maxillofacial Surgery, NIMS Dental College, Jaipur.

\section{NAME ADDRESS EMAIL ID OF THE}

\section{CORRESPONDING AUTHOR:}

Dr. Anjali Wadhwa,

1788, Gau Shala Street,

Muktsar - 152026, Punjab.

E-mail: dr.anjaliwadhwa1987@gmail.com

Date of Submission: 07/03/2014.

Date of Peer Review: 08/03/2014.

Date of Acceptance: 14/03/2014.

Date of Publishing: 27/03/2014. 